\title{
A heterozygous microdeletion of 20q13.13 encompassing ADNP gene in a child with Helsmoortel-van der Aa syndrome
}

\author{
Minh-Tuan Huynh ${ }^{1,2}$ - Elise Boudry-Labis ${ }^{1} \cdot$ Alfred Massard $^{3} \cdot$ Caroline Thuillier $^{1} \cdot$ Bruno Delobel $^{4} \cdot$ \\ Bénédicte Duban-Bedu ${ }^{4} \cdot$ Catherine Vincent-Delorme ${ }^{5}$
}

Received: 8 September 2017 / Revised: 3 April 2018 / Accepted: 11 April 2018 / Published online: 13 June 2018

(c) European Society of Human Genetics 2018

\begin{abstract}
Helsmoortel-van der Aa (SWI/SNF autism-related or $A D N P$ syndrome) is an autosomal dominant monogenic syndrome caused by de novo variants in the last exon of $A D N P$ gene and no deletions have been documented to date. We report the first case of a 3 years and 10 months old boy exhibiting typical features of $A D N P$ syndrome, including intellectual disability, autistic traits, facial dysmorphism, hyperlaxity, mood disorder, behavioral problems, and severe chronic constipation. 60K Agilent array-comparative genomic hybridization (CGH) identified a heterozygous interstitial microdeletion at 20q13.13 chromosome region, encompassing $A D N P$ and $D P M 1$. Taking into account the clinical phenotype of previously reported cases with $A D N P$ single-point variants, genotype-phenotype correlation in the proband was established and the diagnosis of Helsmoortel-van der Aa syndrome was made. Our report thus confirms that $A D N P$ haploinsufficiency is associated with Helsmoortel-van der Aa syndrome as well as highlights the utility of whole-genome array-CGH for detection of unbalanced submicroscopic chromosomal rearrangements in routine clinical setting in patients with unexplained intellectual disability and/or syndromic autism.
\end{abstract}

\section{Introduction}

Helsmoortel-van der Aa (MIM \#615873), a novel recognizable intellectual disability syndrome with autism comorbidity, is a monogenic autosomal dominant disorder caused by de novo frameshift or nonsense sequence alterations in the $3^{\prime}$ end of the last exon of $A D N P$ [1]. All individuals with Helsmoortel-van der Aa syndrome had consistent clinical features, including autism spectrum disorders, mild to severe intellectual disability, and distinctive

Electronic supplementary material The online version of this article (https://doi.org/10.1038/s41431-018-0165-8) contains supplementary material, which is available to authorized users.

Minh-Tuan Huynh

minhtuannia82@yahoo.it

1 Institut de Génétique Médicale et Université de Lille 2, Hôpital Jeanne de Flandre, Lille, France

2 Pham Ngoc Thach, Medical University, Ho Chi Minh city, Vietnam

3 Centre Hospitalier de Cambrai, Cambrai, France

4 Centre de Cytogénétique, Hôpital Saint Vincent de Paul, GHICL, Lille, France

5 Service de Génétique Clinique Guy Fontaine et Université de Lille 2, Hôpital Jeanne de Flandre, Lille, France facial dysmorphism [2]. ADNP (MIM* 611386) is composed of five exons and encodes an activity-dependent neuroprotective homeobox protein. 3D genome structure analysis allows an overview of regulatory landscape and 3D genome structural units within the ADNP locus (Supplemental Figure S1). This Krüppel C2H2-type zinc-finger transcriptional factor is a component of human BAF multiprotein complexes and participates in the regulation of neuronal development through chromatin remodeling [3]. Interstitial deletions of 20q13.13 chromosome region have been rarely documented. To date, only five heterozygous interstitial deletions with different sizes ranging from 313 $\mathrm{kb}$ to $3.31 \mathrm{Mb}$ involving (partial) ADNP gene have been recorded (Table 1 and Fig. 2a). Moreover, no cases with pure $A D N P$ deletions have been reported so far. We report the first case exhibiting the typical features of Helsmoortel-van der Aa syndrome carrying the smallest 63 $\mathrm{kb}$ heterozygous interstitial deletion at 20q13.13 chromosome region. Having reviewed previously reported cases with deleterious single-point variants, the genotype-phenotype correlation of the present index-patient was established.

\section{Materials and methods}

Blood lymphocytes were cultured in RPMI 1640 supplemented with PHA (Life Technologies Sas, Courtaboeuf, 


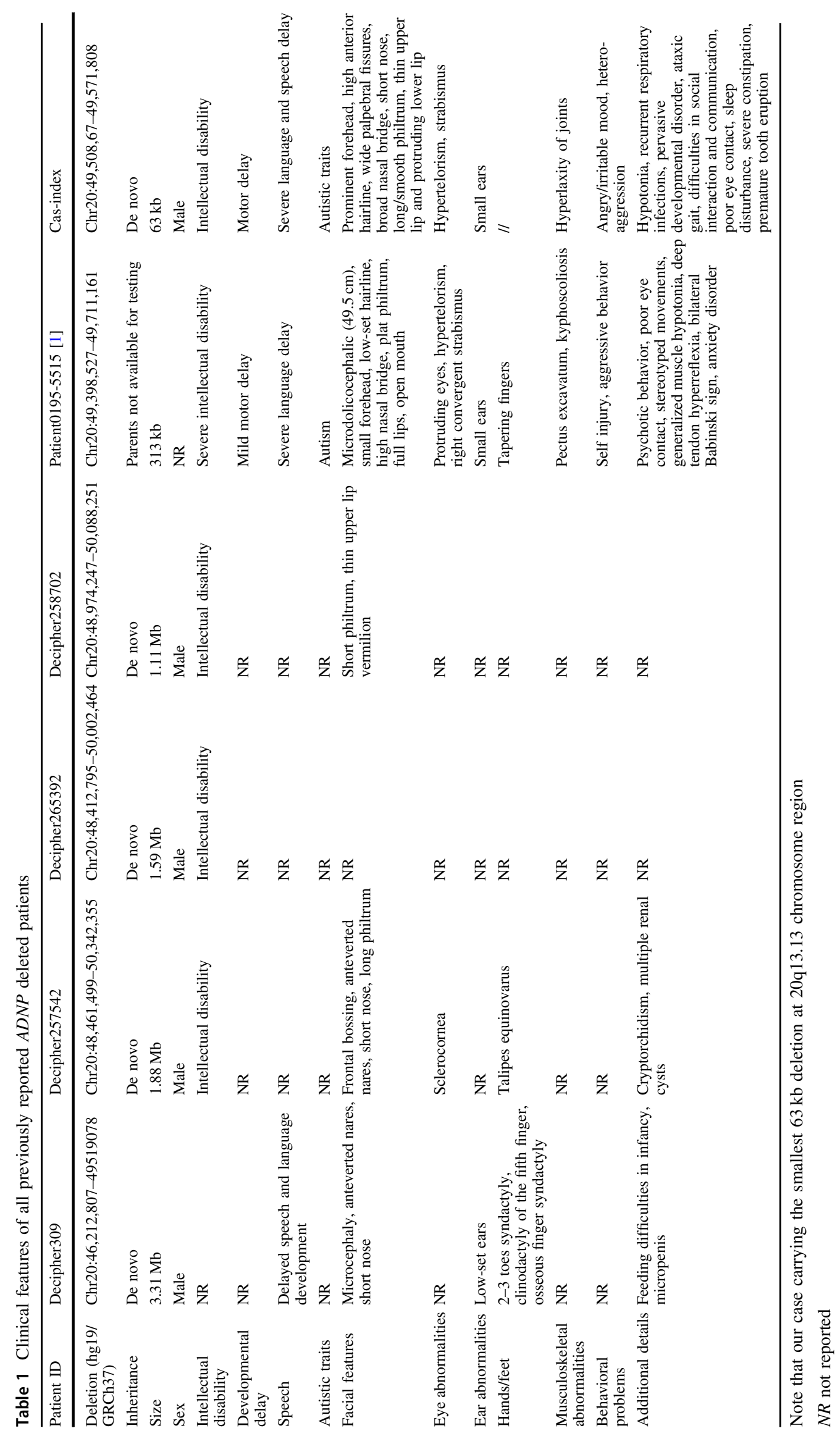


A

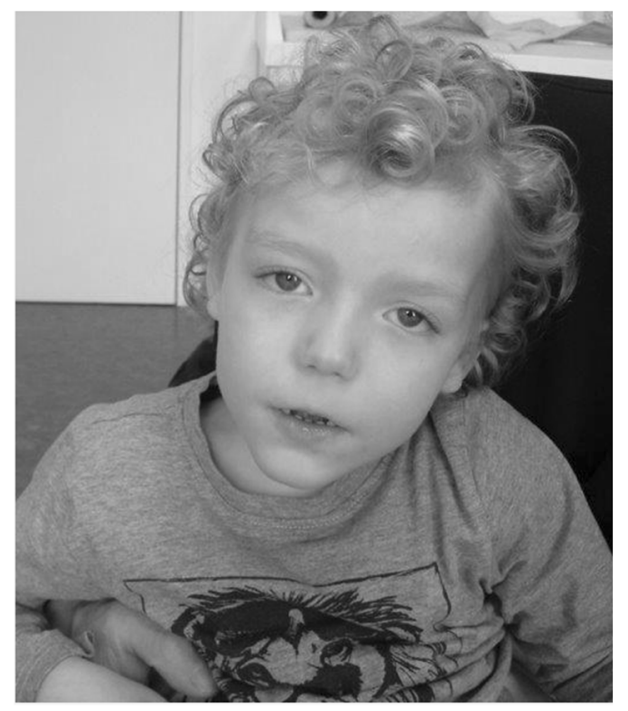

B

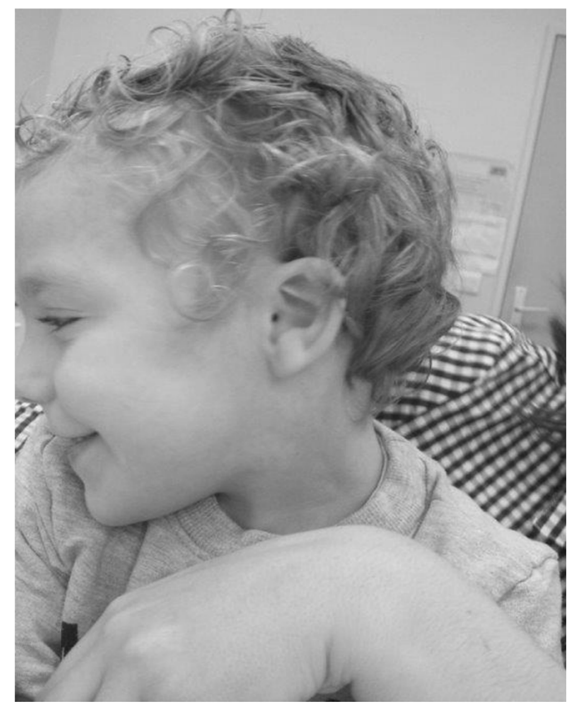

C

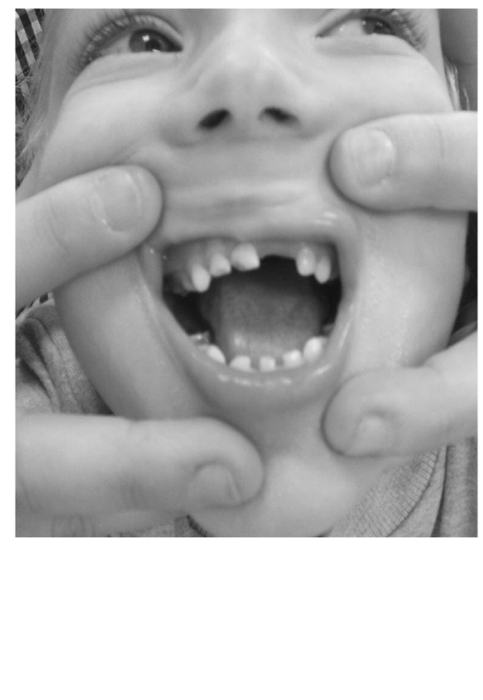

Fig. 1 Photographs of the face $(\mathbf{a}, \mathbf{c})$ and right profile (b) of the proband showed typical facial dysmorphic features, including: frontal bossing, high anterior hairline, broad nasal bridge, hypertelorism, wide

France) and chromosome metaphases were harvested with minor modifications according to the standard laboratory protocol as previously described. Fluorescent in situ hybridization (FISH) analysis was performed using two BAC probes: RP5-914P20 (chr19:49,495,091-49,600,424) and RP11-466J8 (chr19:2,840,928-3,027,437) according to the laboratory protocol [4]. 60K array-CGH (Agilent Technologies, Santa Clara, CA, USA) with a median probe spacing of $54 \mathrm{~kb}$ was carried out as previously described [5] and required at least three contiguous probes to make a call. Quantitative real-time PCR using SYBR $^{\circ}$ Green PCR Master Mix (Life Technologies, Woolston Warrington, UK) was performed and all samples were run in triplicate. The dosage of each amplicon relative to control amplicon of RPPHI (gene ID85495) and normalized to control DNA using the $2-\Delta \Delta \mathrm{CT}$ method as described [6]. Total RNA from blood sample was extracted according to Maxwell 16 IVD instrument Nucleic Acid Extraction-Protocol Promega. One microgram of total RNA was used for reversetranscriptase PCR (Thermo Fisher Scientific, Lithuania). Results were normalized with endogenous control gene $\beta-2$ microglobulin $(B 2 M G)$ as previously described [7].

\section{Results}

\section{Clinical phenotype}

The 3 years and 10 months old boy first came to our clinic because of intellectual disability associated with pervasive developmental disorder. The pregnancy was marked with maternal hyperglycemia. He was born at $39+6$ weeks to a palpebral fissures, short nose, smooth/long philtrum, small and widely spaced teeth, thin upper lip, and protruding lower lip

healthy non-consanguineous Caucasian couple. His birth weight, length, and head circumference were, respectively, $3320 \mathrm{~g}$ (25th-50th centile), $47 \mathrm{~cm}$ (10th-25th centile), and $36 \mathrm{~cm}$ ( $>90$ th centile). Hypotonia was noted at birth. The family history was unremarkable, the couple already had a healthy 11-year-old child. The proband had recurrent respiratory tract infections, he suffered from congenital stridor and obstructive sleep apnea due to tonsillar hypertrophy, and underwent tonsillectomy at the age of 1 year. His developmental milestones were delayed and with kinesitherapy he could walk unsupported at 24 months. According to clinical evaluation conducted at the age of 55 months, his weight, height, and head circumference were $18 \mathrm{~kg}$ ( 75 th centile), $105 \mathrm{~cm}$ (50th centile), and $49.8 \mathrm{~cm}$ (25th centile), respectively. Clinical physical evaluation showed prominent forehead with high anterior hairline, hypertelorism with inner intercanthal distance of $4 \mathrm{~cm}$ (97th centile), strabismus, palpebral fissure length of $30 \mathrm{~mm}$, small ears with an ear length of $4 \mathrm{~cm}$ ( $<3$ th centile), broad nasal bridge, short nose, long/smooth philtrum, thin upper lip and protruding lower lip, hyperlaxity of the joints, and ataxic gait (Fig. 1). He had severe language delay, spoke the first word at 16 months, and combined words into short sentences at 4 years. Of note, the index-patient displayed premature primary tooth eruption (deciduous tooth eruption at the age of 2 months). He had 12 maxillary teeth, 11 mandibular teeth but lacked 1 mandibular incisor. His four 6-year molars were also prematurely present. He had difficulties in social interaction and communication, poor eye contact, and sleep disturbances. Behavioral problems and the presence of episodic mood disorders such as angry, irritable mood, and hetero-aggression were observed. His parents also 
$\mathbf{A}$

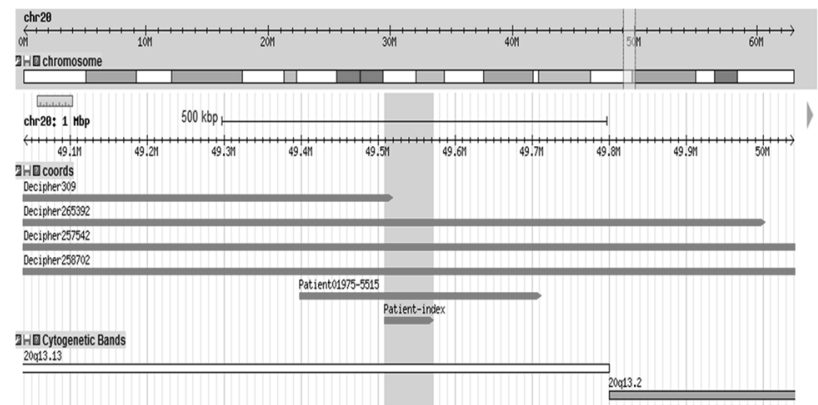

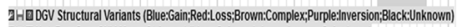
DHQ Nat Rev. Gen. CWV Map. Stringent (blue-Gain; red-Loss; brown=Gaintloss)

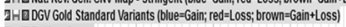

aHaRerSeq Genes

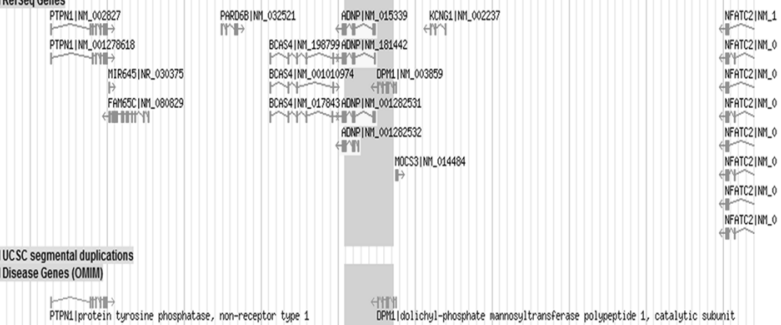

Fig. 2 a Overview of $1 \mathrm{Mb}$ at 20q13.13 chromosome region with five reported overlapping deletions in medical literature: Decipher309, Decipher265392, Decipher257542, Decipher258702, Patient019755515, and index-patient. Gray shadow highlights the smallest region of overlap (SRO); the proband carried the smallest $63 \mathrm{~kb}$ deletion at $20 \mathrm{q} 13.13$ chromosome region harboring two genes $A D N P$ and $D P M 1$. The ADNP gene in Helsmoortel-van der Aa syndrome is highlighted

noticed that he had severe chronic constipation and was treated with laxatives. Echocardiogram, renal ultrasound, chest X-ray, EEG (ElectroEncephaloGram), brain magnetic resonance imaging (MRI), ophthalmologic examination, creatine kinase (CK) dosage, and transferrin isoelectric focusing were normal.

\section{Conventional karyotype and molecular characterization of an interstitial $20 q 13.13$ microdeletion}

Conventional cytogenetics showed a normal male karyotype $46, \mathrm{XY}$. Agilent $60 \mathrm{~K}$ array-CGH analysis showed a heterozygous interstitial deletion at 20q13.13 chromosome with three probes (A_16_P03530666, A_14_P134776, and A_14_P104282), $\log _{2}$ ratio $=-0.85$, encompassing two genes: $A D N P$ and $D P M 1$, chr20:g. $\left(49,457,856 \_49,508,676\right)$ _(49,571,808_49,620,163)del (hg19, NC_000020.10) (NG_034200.1) (Fig. 2b). Quantitative real-time PCR with primers in exons 1 and 5 of $A D N P$ gene did not detect the deletion in the parental DNA (Fig. 2c). FISH analysis showed that the deletion occurred de novo in the proband and excluded parental balanced insertional translocation (Supplemental Figure S2). Quantitative reversetranscriptase PCR showed that ADNP expression was

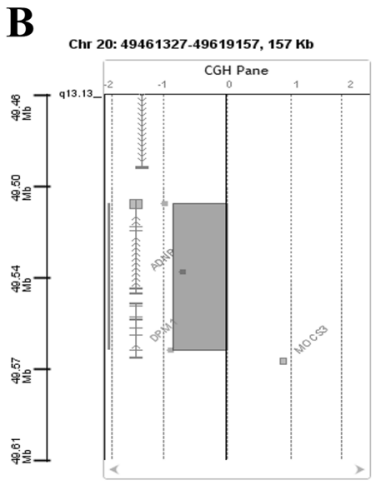

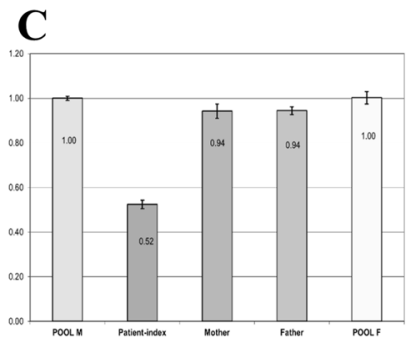

ADNP Exon 1

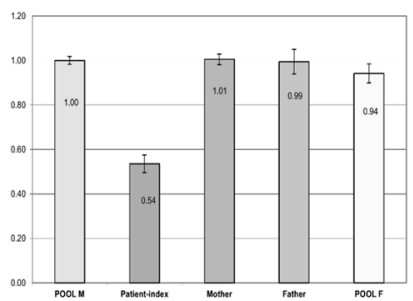

ADNP Exon 5 in yellow. b $60 \mathrm{~K}$ array-CGH showed a $63 \mathrm{~kb}$ interstitial deletion at chromosome 20q13.13 containing ADNP and DPM1. c Quantitative real-time PCR showed that the deletion occurred de novo in the proband with primers in exons 1 and 5 of $A D N P$ gene (index-patient: purple color; mother: pink color; father: blue color; pool of male control: light blue color; pool of female control: yellow color)

decreased approximately $40 \%$ in the proband in comparison to his mother and two normal controls (Supplemental Figure S3).

\section{Discussion}

Hitherto, 18 patients with Helsmoortel-van der Aa syndrome have been reported [8]. Novel additional clinical features associated with $A D N P$ syndrome, including blepharophimosis, craniosynostosis, primary hypothyroidism, hypertension, lack of autistic behaviors, cleft palate, and early deciduous tooth eruption were also recorded [9-11]. All frameshift and nonsense $A D N P$ sequence variations were gain-of-function variants except for a stop-codon variant in the fourth exon with predicted loss of $A D N P$ function. The index-patient carried a de novo $63 \mathrm{~kb}$ heterozygous interstitial deletion at $20 \mathrm{q} 13.13$ chromosome region containing two genes: $A D N P$ and DPM1. The deletion has never been reported in the databases of genomic variants (DGV at http://dgv.tcag.ca/dgv) (Supplemental Figure S4). DPM1 (MIM* 603503) encodes a key enzyme dolichol phosphate mannose (DPM) synthase which plays an important function in protein glycosylation. Diseaseassociated sequence variants of DPMI cause congenital 
disorder of glycosylation type Ie (MIM \#608799). To date, nine reported patients had typical features, including: seizure (8/9), postnatal microcephaly (8/9), abnormal MRI (8/9), and increased serum CK levels (7/9) [12]. Based on the detailed analysis of clinical findings, it is worth noting that the clinical features as well as paraclinical examinations of the reported proband are not consistent with the diagnosis of congenital disorder of glycosylation type Ie. Of interest, the proband displayed typical findings of Helsmoortel-van der Aa syndrome. Additionally, he had severe chronic constipation. Only 4 of 18 reported patients had constipation, one carrying a de novo c.2491_2494delTTAA p.Lys831Ilefs*81 variant had intermittent constipation, while other three patients had the same sequence alteration c. $2157 \mathrm{C}>\mathrm{G}$ p.Tyr719* in $A D N P$ gene. Up to date, all reported disease-associated variants apparently escaped from nonsense-mediated mRNA decay which showed an increased aberrant ADNP mRNA. Unlike dominantnegative mutational mechanism, $A D N P$ loss-of-function variants or deletion would produce an amorphic allele leading to gene dosage imbalance hypothesis. Normal BAF complex function requires the physical interaction of distinct subunits which ideally are mutually balanced. The presence of multiple heterozygous variants in genes encoding different components of BAF complex would lead to synergistic effects that could drive the multimer concentration and/or activity below a critical thresold required for a normal phenotype or otherwise, ADNP haploinsufficiency associated with hypomorphic alleles of other protein partners in BAF complex could cause a reduced complexes activities below a critical thresold resulting in an abnormal phenotype [13]. Moreover, regarding the probability of lossof-function intolerance (pLI) score, $A D N P$ has a high pLI score of 1.0 which is particularly intolerant to loss-of-function variants, dosage sensitive and predicted to have a high probability of being relevant to haploinsufficiency ( $\mathrm{pLI} \geq 0.9$ ), while low $D P M 1$ pLI score of 0 is loss-of-function tolerant. The $A D N P$ gene region on $8 \times 60 \mathrm{~K}$ array-CGH platform is inadequately covered and contains only two probes: A_16_P03530666 and A_14_P134776 which could not detect small intragenic $A D N P$ rearrangements during routine array-CGH testing. High-resolution techniques should be preferentially performed in patients with unexplained intellectual disability in order to tackle these issues [14]. In summary, we report the first case of $A D N P$ haploinsufficiency associated with Helsmoortel-van der Aa syndrome and highlight the importance of high-resolution techniques such as high-density oligonucleotide array-CGH and whole-genome sequencing in clinical diagnostic workup when all available conventional and specific gene tests did not reveal causative abnormalities.

\section{Compliance with ethical standards}

Conflict of interest The authors declare that they have no conflict of interest.

Ethics approval This work is not a clinical research and considered as routine clinical care.

\section{References}

1. Helsmoortel C, Vulto-van Silfhout AT, Coe BP, et al. A SWI/ SNF-related autism syndrome caused by de novo mutations in ADNP. Nat Genet. 2014;46:380-4.

2. Li C, Wang Y, Szybowska M. Novel features of Helsmoortel-Van der Aa/ADNP syndrome in a boy with a known pathogenic mutation in the ADNP gene detected by exome sequencing. Am J Med Genet A. 2017; https://doi.org/10.1002/ajmg.a.38201.

3. Vandeweyer G, Helsmoortel C, van Dijck A, et al. The transcriptional regulator ADNP links BAF (SWI/SNF) complexes with autism. Am J Med Genet C. 2014;166C:315-26.

4. Howe B, Umrigar A, Tsien F. Chromosome preparation from cultured cells. J Vis Exp. 2014;83:e50203.

5. Perry GH, Ben-Dor A, Tsalenko A, Sampas N, RodriguezRevenga L, Tran CW, Scheffer A, Steinfeld I, Tsang P, Yamada NA, Park HS, J1 Kim, Seo JS, Yakhini Z, Laderman S, Bruhn L, Lee C. The fine-scale and complex architecture of human copy-number variation. Am J Hum Genet. 2008;82:685-95.

6. Yuan JS, Wang D, Stewaaaaaart CN,Jr . Statistical methods for efficiency adjusted real-time PCR quantification. Biotechnol $\mathrm{J}$. 2008;3:112-23.

7. Braitch M, Kawabe K, Nyirenda M, Gilles LJ, Robins RA, Gran B, Murphy S, Showe L, Constantinescu CS. Expression of activity-dependent neuroprotective protein in the immune system: possible functions and relevance to multiple sclerosis. Neuroimmunomodulation. 2010;17:120-5.

8. Pescosolido MF, Schwede M, Johnson Harrison A, et al. Expansion of the clinical phenotype associated with mutations in activity-dependent neuroprotective protein. J Med Genet. 2014;51:587-9.

9. Takenouchi T, Miwa T, Sakamoto Y, Sakaguchi Y, Uehara T, Takahashi T, Kosaki K. Further evidence that a blepharophimosis syndrome phenotype is associated with a specific class of mutation in the ADNP gene. Am J Med Genet A. 2017;173:1631-4.

10. Gozes I, van Dijck A, Hacohen-Kleiman G, et al. Premature primary tooth eruption in cognitive/motor-delayed ADNP-mutated children. Transl Psychiatry. 2017;7:e1166.

11. Krajewska-Walasek M, Jurkiewicz D, Piekutowska-Abramczuk D, Kucharczyk M, Jezela-Stanek A, Ciara E. Additional data on the clinical phenotype of Helsmoortel-Van der Aa syndrome associated with a novel truncating mutation in ADNP gene. Am J Med Genet A. 2016;170:1647-50.

12. Bursle C, Brown D, Cardinal J, Connor F, Calvert S, Coman D. DMP1-CDG (CDG1e) with significant gastrointestinal manifestations; phenotype and genotype expansion. JIMD Rep. 2017;34: $27-32$.

13. Veitia RA. A generalized model of gene dosage and dominant negative effects in macromolecular complexes. FASEB J. 2010;24:994-1002.

14. Hochstenbach R, Buizer-Voskamp JE, Vorstman JA, Ophoff RA. Genome arrays for the detection of copy number variations in idiopathic mental retardation, idiopathic generalized epilepsy and neuropsychiatric disorders: lessons for diagnostic workflow and research. Cytogenet Genome Res. 2011;135:174-202. 